\title{
VALORES NORMATIVOS DA APTIDÃO FÍSICA PARA IDOSAS BRASILEIRAS DE 60 A 69 ANOS DE IDADE
}

NORMATIVE VALUES OF PHYSICAL FITNESS FOR BRAZILIAN ELDERLY WOMAN AGED 60-69 YEARS

VALORES NORMATIVOS DE LA APTITUD FISICA PARA MUJERES MAYORES

BRASILEÑAS CON 60 A 69 AÑOS
Giovana Zarpellon Mazo

(Educador Físico)

Daniel Rogério Petreça'

(Educador Físico)

Paula Fabricio Sandreschi'

(Educador Físico)

Tânia Rosane Bertoldo Benedetti² (Educador Físico)

1. Universidade do Estado de Santa Catarina (UDESC), Florianópolis,

SC, Brasil.

2. Universidade Federal de Santa Catarina (UFSC), Florianópolis, SC, Brasil.

\section{Correspondência:}

Universidade do Estado de Santa Catarina (UDESC) Florianópolis, SC, Brasil.

Rua Pascoal Simone, 358, Coqueiros, Florianópolis. 88080-350. SC, Brasil. giovana.mazo@udesc.br

\section{RESUMO}

Introdução: A bateria Senior Fitness Test (SFT) foi difundida e utilizada para a avaliação da aptidão física de idosos de diferentes países. No Brasil, foi aplicada em vários estudos, utilizando-se como padrão de critério os valores de referência americanos para a avaliação, porque no Brasil não há valores desenvolvidos para idosos. Objetivo: Desenvolver valores normativos da bateria Senior Fitness Test (SFT) para idosas brasileiras praticantes de exercícios físicos, na faixa etária de 60 a 69 anos, bem como compará-los com os valores de referência das idosas americanas e portuguesas. Métodos: Foram avaliadas, por meio da bateria SFT, 335 idosas praticantes de exercícios físicos e residentes em Florianópolis, SC, Brasil. A bateria é composta por cinco testes que avaliam força de membros inferiores e superiores, flexibilidade de membros inferiores e superiores, agilidade e equilíbrio dinâmico e resistência aeróbia. Foi utilizada estatística inferencial. Para retirar os outliers foi utilizado o escore $Z$ e, para a obtenção dos valores normativos calcularam-se percentis $\left(P_{5}\right.$ a $\left.P_{95}\right)$. A soma dos percentis foi utilizada para obter o nível de aptidão física para idosos (NAFI). Resultados: Foram estabelecidos valores normativos nas faixas etárias de 60 a 64 anos e 65 a 69 anos e ao comparar os valores criados com já existentes (portugueses e americanos) verificou-se variabilidade na maioria dos percentis, não ocorrendo uma constante em relação ao melhor desempenho. Conclusão: Os resultados permitem estabelecer, por meio do escore-percentil, a possibilidade de obter um NAFI que pode ser utilizado em diversas fases de um programa de exercício físico, desde o seu planejamento até execução e avaliação, bem como um parâmetro para a mudança de comportamento para a prática de exercícios físicos.

Palavras-chave: idoso, aptidão física, atividade motora.

\begin{abstract}
Introduction: Senior Fitness Test (SFT) was widespread and used for assessing the fitness of senior citizens from different countries. In Brazil, it has been applied in several studies, and used as a criterion standard of the American benchmarks for the evaluation, because in Brazil no standards were developed for elderly. Objective: To develop normative values of the Senior Fitness Test (SFT) for Brazilian elderly women practitioners of physical exercises, aged 60-69 years, and compares them with the reference values of US and Portuguese older women. Methods: Using the SFT, 335 elderly women engaged in physical exercises and residents in Florianópolis / SC, Brazil were evaluated. The STF consists of five tests that assess strength of upper and lower limbs, flexibility of upper and lower limbs, agility and dynamic balance and endurance. Inferential statistics was used. To remove the outliers the Z score was used and to obtain the normative values the percentiles (P5 to P95) were calculated. The sum of percentiles was used to obtain the level of fitness for elderly (LFE). Results: Normative values were established in the age groups 60-64 years and 65-69 years and when comparing the values created with those existing (Portuguese and American) variability was found in most percentiles, and there was no constant in relation to better performance. Conclusion: The results indicate through the score-percentile the possibility of obtain a LFE that could be used in various stages of an exercise program, from its planning to implementation and evaluation, as well as a parameter for change behavior for physical exercise.
\end{abstract}

Keywords: aged, physical fitness, motor activity.

\section{RESUMEN}

Introducción: La serie Senior Fitness Test (SFT) fue difundida y utilizada para la evaluación de la aptitud física de personas mayores de diferentes países. En Brasil, fue aplicada en varios estudios, usándose como estándar de criterio los valores de referencia estadounidenses para la evaluación, porque en Brasil no hay valores desarrollados para personas mayores. Objetivo: Desarrollar valores normativos de la serie Senior Fitness Test (SFT) para mujeres mayores brasileñas practicantes de ejercicios físicos, en el grupo de edad de 60 a 69 años, bien como compararlos con los valores de referencia de las mujeres mayores estadounidenses y portuguesas. Métodos: Fueron evaluadas, a través de la serie SFT, 335 mujeres mayores practicantes de ejercicios físicos y residentes en Florianópolis/SC, Brasil. La serie está compuesta por cinco tests que evalúan fuerza de miembros inferiores y superiores, flexibilidad de miembros inferiores y superiores, agilidad y equilibrio dinámico y resistencia aeróbica. Fue utilizada estadís- 
tica inferencial. Para retirar los outliers fue utilizado el score Z y para la obtención de los valores normativos se calcularon percentiles $\left(P_{5}\right.$ a $\left.P_{95}\right)$. La suma de los percentiles fue utilizada para obtener el nivel de aptitud fisica para personas mayores (NAFPM). Resultados: Fueron establecidos valores normativos en los grupos de edad de 60 a 64 años y 65 a 69 años y al comparar los valores creados con los ya existentes (portugueses y estadounidenses) se verificó variabilidad en la mayoría de los percentiles, sin ocurrir una constante en relación al mejor desempeño. Conclusión: Los resultados permiten establecer, por medio del escor-percentil, la posibilidad de obtener un NAFPM que puede ser utilizado en diversas fases de un programa de ejercicios físicos, desde su planificación hasta la ejecución y evaluación, bien como un parámetro para el cambio de comportamiento para la práctica de ejercicios físicos.

Palabras clave: anciano, aptitud física, actividad motriz.

\section{INTRODUÇÃO}

Dentre dos diversos instrumentos utilizados para medir a aptidão física de idosos está a bateria de testes Senior Fitness Test (SFT). A SFT foi desenvolvida e validada para idosos pelas pesquisadoras Jessie Jones e Roberta Rikli da California State University, California, Estados Unidos, como parte do Life Span Assessments Projet ou Fullerton Functional Fitness Test, que teve duração de aproximadamente 6 anos (1994 a 2000) ${ }^{1}$.

Essa bateria de testes mensura a aptidão física de idosos para realizar as atividades da vida diária na velhice e é composta por seis testes motores que avaliam a força dos membros superiores e inferiores, flexibilidade dos membros superiores e inferiores, agilidade/equilíbrio dinâmico e resistência aeróbia de acordo com a faixa etária'.

Além disso, as autoras apresentam valores de normalidade para idosos americanos com idade entre 60 a 94 anos. Esses valores permitem avaliar os padrões de desempenho de acordo com faixa etária (de 5 em 5 anos) e sexo e estão associados com a perda da mobilidade funcional e de independência física na idade avançada².

Diante disso, a SFT foi difundida e utilizada para a avaliação da aptidão física de idosos de diferentes países ${ }^{2-4}$. No Brasil, vários estudos aplicaram a bateria e utilizaram para a avaliação dos idosos como padrão de critério os valores de referência americanas para a avaliação dos idosos ${ }^{5-13}$.

Os valores de normalidade desenvolvidos por Rikli e Jones ${ }^{2}$, conforme Varela et al. ${ }^{14}$ devem ser utilizados com cautela, uma vez que foram baseados em uma amostra relativamente heterogênea e que refletem a população americana.

Neste aspecto, Marques et al. ${ }^{4}$ desenvolveram valores normativos das aptidões físicas avaliadas na SFT para idosos portugueses. Deste modo, observa-se que diante da enorme diversidade e complexidade cultural dentro de países e entre regiões do mundo ${ }^{15}$, o desenvolvimento de valores normativos para idosos brasileiros de determinada cultura, gênero e idade podem contribuir tanto para pesquisa como para aplicação prática, visto que estes padrões tornam possível avaliar o nível de aptidão individual e fornecer um parâmetro para a implementação de estratégias de saúde da população para combater a perda precoce de independência ${ }^{1,4}$.

O objetivo desse estudo foi desenvolver valores normativos da bateria SFT para mulheres idosas brasileiras praticantes de exercícios físicos na faixa etária de 60 a 69 anos, bem como compará-los com os valores de referência dos idosos americanos e portugueses.

\section{MÉTODO}

Esta pesquisa foi aprovada pelo Comitê de Ética para Seres Humanos da Universidade do Estado de Santa Catarina, Florianópolis, SC, Brasil com o registro no 185/2007. Todas as idosas foram informadas sobre o objetivo da pesquisa e assinaram o Termo de Consentimento Livre e Esclarecido.

A amostra deste estudo foi selecionada intencionalmente e composta por 335 mulheres de 60 a 69 anos de idade, participantes de Programas de Exercícios Físicos para idosos do município de Florianópolis, SC, Brasil e que realizavam atividade física sistematizada e supervisionada há pelo menos 6 meses. Todas as idosas eram fisicamente independentes.

\section{Instrumentos e coleta de dados}

A bateria de testes utilizada a fim de obtenção dos valores normativos para mulheres idosas foi a Senior Fitness Test (SFT). Essa bateria é composta por seis testes motores que avaliam a força dos membros superiores (Flexão de antebraço) e inferiores (Levantar e sentar na cadeira), flexibilidade dos membros superiores (Alcançar atrás das costas) e inferiores (Sentar e Alcançar), agilidade/equilíbrio dinâmico (Sentado, caminhar 2,44m e voltar a sentar) e resistência aeróbia (Andar 6 minutos ou marcha estacionária) de acordo com a faixa etária². Rikli e Jones", para avaliar resistência aeróbia, apresenta a opção por um dos testes (andar 6 minutos ou a marcha estacionária de 2 minutos).

A SFT foi validada de acordo com os três tipos de evidências propostas pela American Psyclogical Association ${ }^{16}$ : validade relacionada ao conteúdo, critério e construto. O valor da validade relacionada ao critério encontrado nos diferentes testes em mulheres variou de $r=0.71$ a 0.81 . A confiabilidade foi avaliada por meio de procedimentos de correlação interclasse (R), os valores de $R$ variaram de 0,80 a 0,97.

Optou-se pelo teste andar 6 minutos na avaliação da resistência aeróbia, visto que é o mais utilizado em estudos brasileiros ${ }^{5-13}$.

Os dados foram coletados por avaliadores treinados. Os testes foram aplicados em formato de circuito, seguindo a sequência dos testes da bateria SFT, conforme os protocolos dos testes propostos Rikli e Jones ${ }^{1}$. A duração da bateria de testes foi em média de 15 minutos.

\section{Análise estatística}

Para a análise dos dados foi utilizado estatística descritiva. Para comparação entre as faixas etárias e os testes de aptidão física foi utilizado teste $t$ de Student no caso da distribuição apresentar normalidade ou Mann Whitney, no caso de distribuição não paramétrica. Procedeu-se a execução de escores $Z$ dos dados para o tratamento de retirada de outliers, definindo um processo de duas etapas: a primeira consistiu na conferência dos dados discrepantes quanto à precisão, realizando a confirmação nas fichas de anotação individual; a segunda ocorreu com a exclusão dos valores, para tal, foi utilizado pontos de corte para o escore $Z$ de \pm 2 . Para a construção da tabela de valores normativos da SFT, utilizou-se o cálculo de percentis $\left(P_{5}, P_{10}, P_{15}, P_{20}, P_{25}, P_{30}, P_{35}, P_{40}\right.$, $\left.P_{45}, P_{50}, P_{55}, P_{60}, P_{65}, P_{70}, P_{75}, P_{80}, P_{85}, P_{90}, P_{95}\right)$ em cada um dos testes. $A$ fim de obter um nível de aptidão física para idosos (NAFI), foi criado uma pontuação de acordo com as aptidões físicas decorrente ao coeficiente obtido individualmente em cada variável como um somatório das capacidades físicas do indivíduo. Para todas as análises foi adotado nível de significância p-valor<0,05. 


\section{RESULTADOS}

A amostra deste estudo foi composta por 335 idosas praticantes de exercício físico com média de idade 64,5 (DP=2,7) anos. O grupo foi dividido em faixas etárias, seguindo o procedimento utilizado originalmente pelos autores da bateria SFT. Sendo assim, 166 (49,6\%) idosas se encontram na faixa etária de 60 a 64 com média de idade 62,0 (DP=1,3) anos e $169(50,4 \%)$ idosas na faixa etária de 65 a 69 com média de idade 66,8 (DP=1,4) anos.

A tabela 1 apresenta os valores de média, mediana, desvio padrão e intervalo de confiança e diferenças entre as faixas etárias dos testes referentes às aptidões físicas.

As tabelas 2 e 3 apresentam os resultados dos 5 testes e seus respectivos percentis de acordo com as faixas etárias.

Tabela 1. Valores de mediana obtidos nos testes da bateria SFT de acordo com as faixas etárias.

\begin{tabular}{c|c|c|c|c|c}
\hline Variável & \multicolumn{2}{|c|}{$60-64$} & \multicolumn{2}{c|}{$65-69$} & p \\
\hline & $\begin{array}{c}\text { Mediana } \\
\text { (DP) }\end{array}$ & $\begin{array}{c}\text { Média } \\
\text { (DP) }\end{array}$ & $\begin{array}{c}\text { Mediana } \\
\text { (DP) }\end{array}$ & $\begin{array}{c}\text { Média } \\
\text { (DP) }\end{array}$ & \\
\hline $\begin{array}{c}\text { Sentar e levantar da } \\
\text { cadeira }\end{array}$ & $15(2,8)$ & $14,9(2,8)$ & $16(3,1)$ & $15,6(3,1)$ & 0,72 \\
\hline Flexão antebraço (d) & $18(3,6)$ & $17,9(3,6)$ & $18(3,8)$ & $18,2(3,8)$ & 0,51 \\
\hline Sentar e alcançar & $3(7,7)$ & $4,8(7,7)$ & $2(8,9)$ & $2,4(8,9)$ & $0,02^{*}$ \\
\hline $\begin{array}{c}\text { Agilidade e equilíbrio } \\
\text { dinâmico }\end{array}$ & $5,4(0,8)$ & $5,4(0,8)$ & $5,54(0,7)$ & $5,5(0,7)$ & 0,28 \\
\hline $\begin{array}{c}\text { Alcançar atrás das } \\
\text { costas (d) }\end{array}$ & $-4(7,4)$ & $-3,6(7,4)$ & $4,5(8,1)$ & $-5,6(8,1)$ & $0,04^{*}$ \\
\hline $\begin{array}{c}\text { Caminhada de } \\
6 \text { minutos }\end{array}$ & $\begin{array}{c}541,5 \\
(56,5)\end{array}$ & $\begin{array}{c}540,6 \\
(56,5)\end{array}$ & $\begin{array}{c}535 \\
(57,6)\end{array}$ & $\begin{array}{c}530 \\
(57,6)\end{array}$ & 0,21 \\
\hline
\end{tabular}

* =nivel de significância pelo teste de Mann_Whitney; DP = desvio padrão; (d) = dominante; $¥$ = valor verificado pelo teste não-paramétrico de acordo com as medianas.

Tabela 2. Valores normativos da bateria SFT baseados no cálculo de percentis, de mulheres ativas entre 60 e 64 anos.

\begin{tabular}{|c|c|c|c|c|c|c|}
\hline Percentis & $\begin{array}{c}\text { Sentar e } \\
\text { levantar } \\
\text { da cadeira } \\
n=156\end{array}$ & $\begin{array}{c}\text { Flexão } \\
\text { antebraço } \\
n=153\end{array}$ & $\begin{array}{c}\text { Sentar e } \\
\text { alcançar } \\
\text { n=152 }\end{array}$ & $\begin{array}{c}\text { Agilidade } \\
\text { e } \\
\text { equilíbrio } \\
\text { dinâmico } \\
n=153\end{array}$ & $\begin{array}{c}\text { Alcançar } \\
\text { atrás das } \\
\text { costas } \\
n=155\end{array}$ & $\begin{array}{c}\text { Caminhada } \\
\text { de } 6 \text { min. } \\
n=154\end{array}$ \\
\hline P95 & 20,00 & 24,00 & 20,00 & 4,19 & 7,60 & 640,00 \\
\hline P90 & 19,00 & 23,00 & 15,700 & 4,38 & 6,00 & 617,00 \\
\hline P85 & 18,00 & 22,00 & 14,00 & 4,54 & 4,00 & 600,00 \\
\hline P80 & 17,00 & 20,00 & 8,00 & 4,76 & 1,60 & 570,00 \\
\hline P75 & 17,00 & 20,00 & 11,00 & 4,91 & 2,00 & 575,00 \\
\hline P70 & 17,00 & 21,00 & 12,00 & 5,00 & 3,00 & 585,00 \\
\hline P65 & 16,00 & 18,70 & 4,00 & 5,11 & $-2,00$ & 550,00 \\
\hline P60 & 16,00 & 19,00 & 5,00 & 5,23 & $-1,00$ & 550,00 \\
\hline P55 & 16,00 & 19,00 & 6,00 & 5,35 & 1,00 & 561,50 \\
\hline P50 & 15,00 & 17,30 & 2,85 & 5,44 & $-5,00$ & 535,00 \\
\hline P45 & 15,00 & 18,00 & 3,00 & 5,50 & $-4,00$ & 541,50 \\
\hline P40 & 14,00 & 16,00 & 0,00 & 5,63 & $-8,00$ & 517,00 \\
\hline P35 & 14,00 & 16,90 & 1,00 & 5,80 & $-7,00$ & 520,75 \\
\hline P30 & 14,00 & 17,00 & 2,00 & 5,90 & $-6,00$ & 530,00 \\
\hline P25 & 13,00 & 15,00 & 0,00 & 6,00 & $-9,00$ & 511,00 \\
\hline P20 & 12,00 & 14,00 & $-1,00$ & 6,16 & $-12,00$ & 480,25 \\
\hline P15 & 12,00 & 15,00 & 0,00 & 6,30 & $-10,00$ & 500,00 \\
\hline P10 & 11,00 & 13,00 & $-2,70$ & 6,52 & $-14,00$ & 467,00 \\
\hline P5 & 10,00 & 11,00 & $-6,00$ & 7,02 & $-17,10$ & 435,00 \\
\hline
\end{tabular}

$\mathrm{O}$ n. apresentou valores diferentes decorrentes à retirada dos scores $Z$ definidos como excluídos e indivíduos que não conseguiram realizar o teste de forma correta.
Tabela 3. Valores normativos da bateria SFT baseados no cálculo de percentis, de mulheres ativas entre 65 e 69 anos.

\begin{tabular}{c|c|c|c|c|c|c}
\hline Percentis & $\begin{array}{c}\text { Sentar e } \\
\text { levantar } \\
\text { da cadeira } \\
\mathbf{n = 1 6 0}\end{array}$ & $\begin{array}{c}\text { Flexão } \\
\text { antebraço } \\
\mathbf{n = 1 5 9}\end{array}$ & $\begin{array}{c}\text { Sentar e } \\
\text { alcançar } \\
\mathbf{n = 1 6 0}\end{array}$ & $\begin{array}{c}\text { Agilidade } \\
\mathbf{e} \\
\text { equilíbrio } \\
\text { dinâmico } \\
\mathbf{n}=\mathbf{1 4 8}\end{array}$ & $\begin{array}{c}\text { Alcançar } \\
\text { atrás das } \\
\text { costas } \\
\mathbf{n = 1 6 5}\end{array}$ & $\begin{array}{c}\text { Caminhada } \\
\text { de 6 min. } \\
\mathbf{n = 1 4 8}\end{array}$ \\
\hline P95 & 20,00 & 25,00 & 16,95 & 4,43 & 6,00 & 612,10 \\
\hline P90 & 19,90 & 24,00 & 15,00 & 4,62 & 3,70 & 600,50 \\
\hline P85 & 19,00 & 23,00 & 13,00 & 4,87 & 2,55 & 593,25 \\
\hline P80 & 18,00 & 20,00 & 8,00 & 4,95 & 1,00 & 575,00 \\
\hline P75 & 18,00 & 22,00 & 11,80 & 5,02 & 1,90 & 579,20 \\
\hline P70 & 17,70 & 20,00 & 7,00 & 5,10 & 0,00 & 565,00 \\
\hline P65 & 17,00 & 19,00 & 5,00 & 5,22 & $-1,70$ & 550,00 \\
\hline P60 & 17,00 & 20,00 & 6,00 & 5,29 & $-2,50$ & 555,00 \\
\hline P55 & 16,00 & 18,00 & 2,00 & 5,40 & $-4,50$ & 535,00 \\
\hline P50 & 16,00 & 19,00 & 3,00 & 5,54 & $-3,35$ & 545,00 \\
\hline P45 & 15,00 & 17,00 & 0,00 & 5,60 & $-7,80$ & 521,20 \\
\hline P40 & 15,00 & 18,00 & 0,50 & 5,70 & $-6,00$ & 530,00 \\
\hline P35 & 14,00 & 16,00 & $-1,00$ & 5,79 & $-10,00$ & 505,00 \\
\hline P30 & 14,00 & 16,00 & $-1,50$ & 5,90 & $-9,00$ & 510,30 \\
\hline P25 & 13,00 & 15,00 & $-5,00$ & 6,00 & $-13,40$ & 475,00 \\
\hline P20 & 13,00 & 16,00 & $-3,37$ & 6,20 & $-12,00$ & 494,00 \\
\hline P15 & 12,15 & 14,00 & $-7,00$ & 6,48 & $-15,50$ & 461,75 \\
\hline P10 & 11,00 & 13,00 & $-9,90$ & 6,80 & $-17,20$ & 450,00 \\
\hline P5 & 10,00 & 12,00 & $-13,00$ & 7,25 & $-20,00$ & 425,60 \\
\hline P25
\end{tabular}

$\mathrm{O}$ n. apresentou valores diferentes decorrentes à retirada dos scores $\mathrm{Z}$ definidos como excluídos e indivíduos que não conseguiram realizar o teste de forma correta.

Com base nos resultados correspondentes aos percentis, foram utilizados como pontos de corte: abaixo do $\mathrm{P}_{10}$ "muito fraco", $\mathrm{P}_{10}$ a $\mathrm{P}_{25}$ "fraco", $P_{25}$ a $P_{75}$ "Regular", $P_{75}$ a $P_{95}$ "Bom" e acima do $P_{90}$ "muito bom".

A tabela 4 apresenta a pontuação que deve ser atribuída ao indivíduo de acordo com seu desempenho em cada teste. Os testes foram agrupados de acordo com sua característica de aptidão física: força (sentar e levantar da cadeira e flexão antebraço), flexibilidade (sentar e alcançar e alcançar atrás das costas), resistência aeróbia (caminhada de 6 minutos) e agilidade e equilíbrio dinâmico (sentado, caminhar 2,44 m e voltar a sentar).

O somatório dos escores-percentis de cada teste representa o nível de aptidão física para idosos (NAFI) que pode ser visualizado quadro 1.

Tabela 4. Valores de pontuação para cada teste da bateria SFT de idosas de 60 a 69 anos de acordo com a classificação.

\begin{tabular}{c|c|c|c|c|c|c|c}
\hline $\begin{array}{c}\text { Classifi- } \\
\text { cação }\end{array}$ & Faixa & $\begin{array}{c}\text { Sentar e } \\
\text { levantar } \\
\text { da } \\
\text { cadeira }\end{array}$ & $\begin{array}{c}\text { Flexão } \\
\text { antebraço }\end{array}$ & $\begin{array}{c}\text { Sentar e } \\
\text { alcançar }\end{array}$ & $\begin{array}{c}\text { Agilidade } \\
\text { e } \\
\text { equilíbrio } \\
\text { dinâmico }\end{array}$ & $\begin{array}{c}\text { Alcançar } \\
\text { atrás } \\
\text { das } \\
\text { costas }\end{array}$ & $\begin{array}{c}\text { Cami- } \\
\text { nhada } \\
\text { de } \\
\text { 6 min. }\end{array}$ \\
\hline $\begin{array}{c}\text { Muito } \\
\text { bom }\end{array}$ & $\begin{array}{c}\text { Acima } \\
\text { do P90 }\end{array}$ & 12,5 & 12,5 & 12,5 & 25,0 & 12,5 & 25,0 \\
\hline Bom & P75 a P90 & 10,0 & 10,0 & 10,0 & 20,0 & 10,0 & 20,0 \\
\hline Regular & $\begin{array}{c}\text { P25 A } \\
\text { P75 }\end{array}$ & 7,50 & 7,50 & 7,50 & 15,0 & 7,5 & 15,0 \\
\hline Fraco & P10 a P25 & 5,0 & 5,0 & 5,0 & 10,0 & 5,0 & 10,0 \\
\hline $\begin{array}{c}\text { Muito } \\
\text { fraco }\end{array}$ & $\begin{array}{c}\text { Abaixo } \\
\text { do P10 }\end{array}$ & 2,5 & 2,5 & 2,5 & 5,0 & 2,5 & 5,0 \\
\hline
\end{tabular}

Quadro 1. Classificação dos testes de acordo com o somatório referentes aos pontos obtidos em cada teste da bateria da SFT.

\begin{tabular}{|c|c|}
\hline Classificação & Pontuação \\
\hline Muito bom & $80-100$ \\
\hline Bom & $60-79$ \\
\hline Regular & $40-59$ \\
\hline Fraco & $20-39$ \\
\hline Muito fraco & $0-19$ \\
\hline
\end{tabular}


A figura 1a-f compara os pontos de corte estabelecidos nesse estudo para os idosos na faixa etária de 60 a 64 em relação aos valores apresentados para idosos americanos².
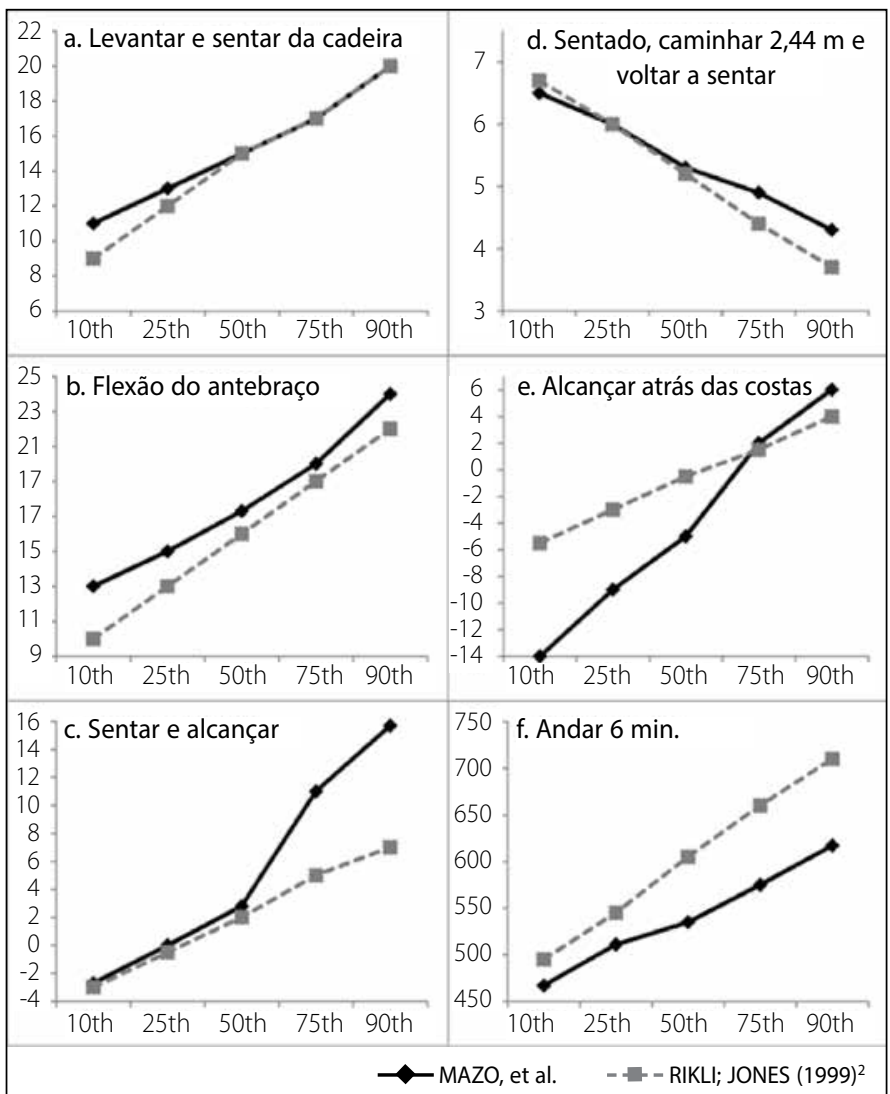

Figura 1a-f. Apresentação da comparação dos pontos de corte de idosos ativos brasileiros com idosos americanos na faixa etária de 60 a 64 anos.

Com exceção dos pontos de corte $\mathrm{P}_{50}$ a $\mathrm{P}_{90}$ no "Levantar e sentar da cadeira" (figura 2a), os outros testes apresentaram valores diferentes. No teste de "Flexão de braço" (figura 2b), os idosos brasileiros foram em média 13,5\% melhores do que os americanos. No teste "Sentar e Alcançar" (figura 2c) os brasileiros obtiveram diferenças a partir do $P_{50}$, chegando a $94 \%$.

No teste "Alcançar atrás das costas" (figura 2e) os idosos brasileiros foram entre $P_{10}$ e $P_{50}, 327 \%$ melhores do que os americanos, melhora essa, invertida nos $\mathrm{P}_{75}$ e $\mathrm{P}_{90}$ chegando a 177\%. Essa melhora dos idosos americanos é vista também no teste de resistência aeróbia "Andar 6 min." (figura 2f), o qual chega a 10\% em comparação aos brasileiros.

A figura 2 compara os pontos de corte estabelecidos nesse estudo para os idosos na faixa etária de 65 a 69 em relação aos valores apresentados para idosos americanos ${ }^{2}$ e portugueses ${ }^{4}$.

$\mathrm{Na}$ faixa etária de 65 a 69 anos, foi incluído como fator de comparação, além dos valores normativos para idosos americanos ${ }^{2}$, os apresentados por Marques et al. ${ }^{4} \mathrm{com}$ idosos portugueses.

Nos testes "Levantar e sentar" e "Flexão de antebraço" (figura 2a,b) idosos brasileiros obtiveram melhor desempenho, exceto no $\mathrm{P}_{90}$ quando os portugueses se sobressaíram aos brasileiros e americanos no $P_{90}$ em 4\% e 16\%; 5,3\% e 14,3\%, respectivamente.

Os idosos portugueses foram inferiores no teste"Sentado, caminhar 2,44 m e voltar a sentar" em 25,3\% em relação aos idosos brasileiros no $P_{10}$. A maior diferença foi verificada no teste de "Alcançar atrás das costas", no qual idosos americanos foram superiores em 322\% e 419\% em relação aos brasileiros e portugueses.

O mesmo ocorreu na faixa etária de 60 a 64 anos no teste de "Caminhada de 6 minutos" (figura 2f) que foi verificado que americanos foram superiores em 29,5\% em relação aos idosos brasileiros e 87\% aos portugueses.

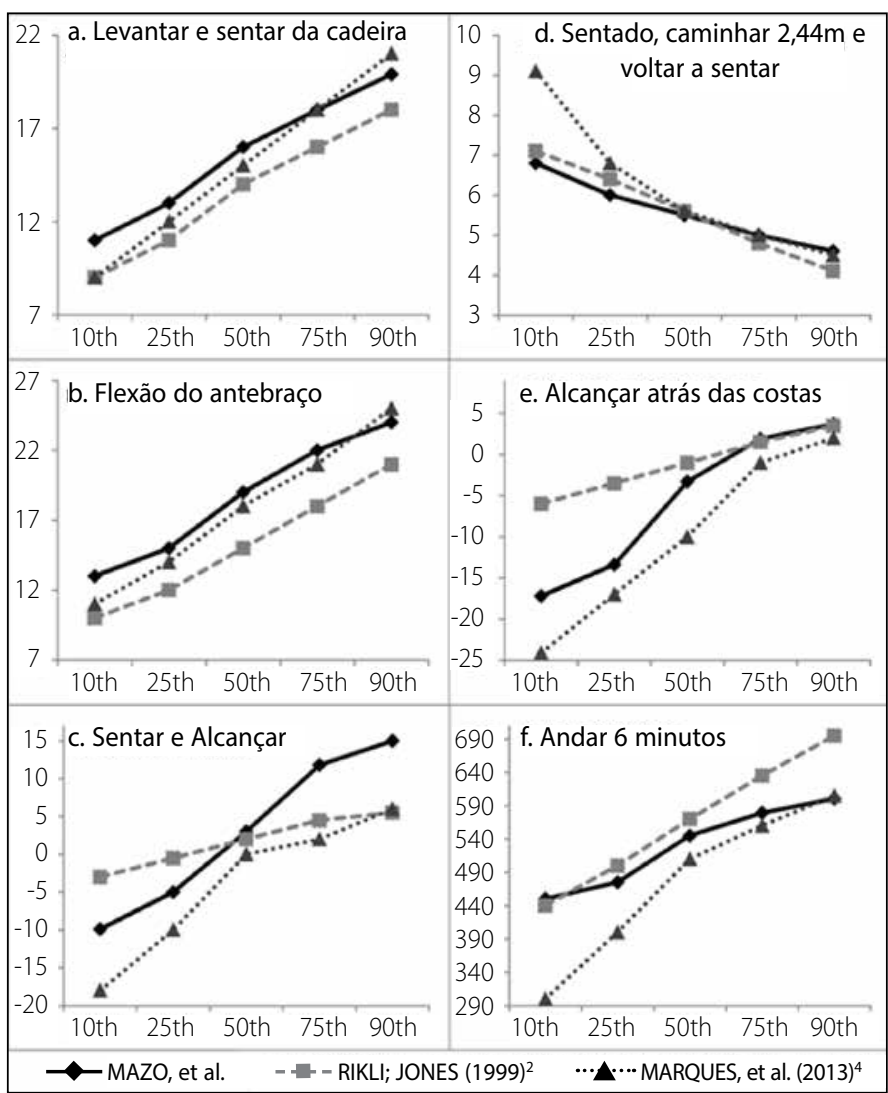

Figura 2a-f. Apresentação da comparação dos pontos de corte de idosos ativos brasileiros com idosos americanos e portugueses na faixa etária de 65 a 69 anos.

\section{DISCUSSÃO}

Este estudo apresenta os valores normativos dos testes da bateria Senior Fitness Test (SFT) para mulheres idosas praticantes de exercícios físicos, no qual foi calculado os valores de percentis, medianas e médias para as faixas de 60 a 64 e 65 a 69 anos de idade.

Ao comparar os resultados das faixas etárias do presente estudo, os testes que mensuram a flexibilidade - tanto de membros inferiores quanto superiores - foram os únicos que apresentaram diferença estatisticamente significativa. Observa-se que isto pode ter ocorrido, porque o envelhecimento acarreta a diminuição da mobilidade articular e da elasticidade muscular que são refletidas na flexibilidade ${ }^{17}$ e a atividade física demonstra ser um componente importante para preservação ou aumento da amplitude das articulações, facilitando a realização de tarefas ${ }^{18}$.

Também essa diferença pode ser reflexo do tipo de exercício físico praticado por cada idosa; uma vez que as capacidades físicas podem responder de forma diferente dependendo do estímulo a qual o sujeito é submetido. Dessa forma, as idosas na faixa de 60 a 69 anos demonstraram serem menos homogêneas em relação à flexibilidade do que referente a outras aptidões físicas.

Ao comparar os valores normativos das aptidões físicas avaliadas na SFT para idosas americanos ${ }^{2}$ e do presente estudo no estrato etário de 60 a 64 anos e incluindo idosas portuguesas ${ }^{4}$ na faixa etária de 65 a 69 anos, foi verificada variabilidade na maioria dos percentis, não ocorrendo uma constante em relação ao melhor desempenho. Tanto no estudo português como no americanos a amostra foi composta por idosas funcionalmente independentes, com participação de 839 e 852 mulheres, respectivamente. No presente estudo além de serem funcionalmente independentes eram praticantes de exercícios físicos.

Nesse sentido, Varela et al. ${ }^{14}$ aconselham que os valores desenvolvidos por Rikli e Jones ${ }^{2}$ foram baseados em uma amostra relativamente heterogênea. Fica assim evidente que a criação de valores 
que refletem a população americana, como especificidade, nível de independência, atividade física e localidade são fundamentais para a identificação mais precisa do diagnóstico do indivíduo em relação a seu nível de aptidão física.

Outro estudo ${ }^{19}$ realizado com 1.033 idosas de Curitiba, PR, Brasil, também comparou o desempenho na bateria SFT (exceto o teste de alcançar atrás das costas) com idosos americanos avaliados por Rikli e Jones ${ }^{2}$. Com relação às mulheres em ambas as faixas etárias, o meIhor desempenho variou sempre entre as idosas avaliadas no presente estudo e as americanas. Ao comparar, em média, os resultados do presente estudo com os das idosos curitibanas, constatou-se que as residentes em Florianópolis,SC apresentaram melhor desempenho em todos os testes, exceto o que avalia flexibilidade de membros inferiores.

Portanto, acredita-se que o desenvolvimento dos escores apresentados contribua para a avaliação e consequente prescrição de exercícios físicos para idosas. A referida bateria de testes pode ser analisada tanto individualmente (prescrição individual de exercícios físicos decorrente situação atual) como coletivamente (análise de um grupo, de um programa ou avaliação de efetividade de uma intervenção) o que ratifica a importância da construção destes valores para mulheres ativas fisicamente. Ressaltando a necessidade de desenvolvimento de escores para as outras faixas etárias e para homens.

Considera-se como limitação deste estudo o estabelecimento de escores normativos com base em amostras de tamanhos desiguais para os testes componentes da bateria

\section{CONCLUSÃO}

Foram estabelecidos valores de normalidade da bateria de testes Senior Fitness Test para idosas brasileiras praticantes de atividade física de 60 a 69 anos de idade, fornecendo assim, um meio disponível para a avaliação da aptidão física nessa população. Além disso, verificou-se as diferenças entre os valores normativos estabelecidos para idosas portuguesas, americanas e brasileiras, verificando assim heterogeneidade nos desempenhos, demostrando a importância para a análise futura de tendência nacional e comparações internacionais. Os resultados permitem estabelecer, por meio do escore-percentil a possibilidade de obter um nível de aptidão física para idosos (NAFI) podendo ser utilizado em diversas fases de um programa de exercício físico, desde o seu planejamento, execução a avaliação, bem como um parâmetro para a mudança de comportamento para a prática de atividade física.

Sugere-se a ampliação deste estudo para outros estratos etários e ambos sexos na diferentes regiões do Brasil.

\section{AGRADECIMENTOS}

Ao Conselho Nacional de Desenvolvimento Científico e Tecnológico - CNPq - pela bolsa de produtividade em pesquisa destinada à orientadora deste estudo Giovana Zarpellon Mazo.

Todos os autores declararam não haver qualquer potencial conflito de interesses referente a este artigo.

\section{REFERÊNCIAS}

1. Rikli R, Jones C. Development and validation of a functional fitness test for community residing older adults. J Aging Phys Act. 1999;7(2):129-61.

2. Rikli $\mathrm{R}$, Jones $\mathrm{C}$. Functional fitness normative scores for community residing older adults, age 60-94. J Aging Phys Act. 1999;7(2):162-81

3. Fournier J, Vuilleminb A, Le Crenc F. Measuring physical fitness in the elderly. Assessment of physical fitness in elderly: French adaptation of the "Senior Fitness Test". Science \& Sports. 2012;27(4):254-59.

4. Marques EA, Baptista F, Santos R, Vale S, Santos DA, Silva AM, et al. Normative functional fitness standards and trends of Portuguese older adults: cross-cultural comparisons. J Aging Phys Act. 2014;22(1):126-37.

5. Nunes MES, Santos $S$. Avaliação funcional de idosos em três programas de atividade física: caminhada, hidroginástica e Lian Gong. Rev Port Cienc Desp. 2009; 9(2-3):150-9.

6. Pereira FD, Batista WO, Furtado HL, Alves Junior ED, Giani TS, Dantas EHM. Comparação da força funcional de membros inferiores e superiores entre idosas fisicamente ativas e sedentárias. Rev Bras Geriatr Gerontol. 2009;12(3):417-27.

7. Sousa LGO, Bueno Júnior CR, Sant'Ana MC, Moura MA. Estudo transversal de variáveis antropométricas e da aptidão física de mulheres idosas de Recife - PE. Rev Mackenzie Educ Fis Esporte. 2010;9(2):121-33.

8. Pereira FD, Batista, WO, Furtado HL, Silva EB, Alves Júnior ED. Funcional autonomy in elderly women who are physically active and sedentary: causal comparative study. Online Braz I Nurs. 2011;10(3):1-10.

9. Vieira LCR, Sousa DS, Silveira MSN, Domiciano TR, Gouvêa-e-Silva LF. Efeito de um programa de treinamento resistido complementar no perfil antropométrico e nas capacidades motoras de mulheres ativas. Braz J Biomotricity. 2011;5(1):16-25
10. Fernandes S, Guimarães ACA, Machado Z, Simas JPN. Flexibilidade de cardiopatas praticantes e não praticantes de atividade física. Rev Bras Ciênc Saúde. 2011;14(4):34-44.

11. Streit IA, Mazo GZ, Virtuoso JF, Menezes EC, Gonçalves E. Aptidão física e ocorrência de quedas em idosos praticantes de exercícios físicos. Rev Bras Ativ Fis Saúde. 2011;16(4):346-52.

12. Elias RGM, Gonçalves ECA, Moraes ACF, Moreira CF, Fernandes CAM. Aptidão física funcional de idosos praticantes de hidroginástica. Rev Bras Geriatr Gerontol. 2012;15(1):79-86.

13. Vila CP, Silva MEM, Simas JPN, Guimarães ACA, Parcias, SR. Aptidão funcional e nível de atenção em idosas praticantes de exercícios físicos. Rev Bras Geriatr Gerontol. 2013;16(2):355-64.

14. Varela S, Ayán C, Cancela J M. Batteries assessing health related fitness in the elderly: a brief review. Eur Rev Aging Phys Act. 2008;5(2):97-105.

15. Organização Mundial da Saúde. Envelhecimento ativo: uma política de saúde. Brasília: Organização Pan-Americana da Saúde; 2005

16. The American Psycological Association. Standards for educational and psychological tests. Washington: American Psychological Association; 1985

17. Dantas EHM, Pereira SAM, Bezerra JCP, Ota AH. A preponderância da diminuicao da mobilidade articula ou da elasticidade muscular na perda da flexibilidade no envelhecimento. Fit Perf J. 2002;1 (3):12-20.

18. Almeida APP, Veras RP, Doimo LA. Avaliação do equilíbrio estático e dinâmico de idosas praticantes de hidroginástica e ginástica. Rev Bras Cineantropom Desempenho Hum. 2010;12(1):55-61.

19. Krause MP, Januário RS, Hallage T, Haile L, Miculis CP, Gama MP, et al. A comparison of functional fitness of older Brazilian and American women. J Aging Phys Act. 2009;17(4):387-97. 